\title{
Spatial recall improved by retrieval enactment
}

\author{
GREGORY V. JONES \\ University of Warwick, Coventry, England \\ AND \\ Maryanne Martin \\ University of Oxford, Oxford, England
}

\begin{abstract}
Evidence from studies of intentional learning suggests that the accuracy of recall is not assisted by appropriate enactment at retrieval, as opposed to encoding. In the present study, long-term recall of spatial arrays following incidental learning (text messaging or calculator use) was tested under three different motor conditions at retrieval. For both letter and number arrays, the accuracy of recall was found to be improved by relevant enactment at the time of retrieval, relative to retrieval with no movement. In contrast, irrelevant movement was found to produce an impairment in accuracy. The overall accuracy of recalling a letter array was found to be a power-law function of the frequency of exposure to the array. The findings are discussed in terms of the hypothesis that appropriate movement during memory retrieval recruits egocentric representations that supplement allocentric representations subserving longer term spatial recall.
\end{abstract}

Early studies of human memory (e.g., Atkinson \& Shiffrin, 1968) were in the forefront of psychology's paradigm shift from behaviorist to cognitivist explanation. One legacy of this may have been to discourage exploration of the possibility that overt behavior - that is, physical movement - can influence the operation of human memory. Nevertheless, evidence has been provided within the working memory framework that irrelevant physical movement may disrupt the initial encoding of spatial information (see Logie, 2003), and it has also been shown conversely that recognition of spatial arrays can be facilitated when they are appropriately pointed at during encoding (Chum, Bekkering, Dodd, \& Pratt, 2007). The further question of whether the recall of previously stored information can be influenced by movement at the time of retrieval has been relatively little investigated, although a notable exception is provided by the subject-performed task (SPT) paradigm.

In the SPT method, participants receive simple sentences (e.g., fold the paper), which they either do or do not also enact. Research has demonstrated that subsequent recall of such sentences is enhanced when the sentences have been enacted at encoding (for a review, see Nilsson, 2000). Noice and Noice (2001) have shown that a similar result occurs if actors learn their lines while moving according to stage directions rather than remaining static, even though their movements bear a less close relation to the text. However, in a particularly relevant study, Kormi-Nouri, Nyberg, and Nilsson (1994) found that there was no further advantage from participants' enacting also at retrieval. In the absence of such an advantage, Kormi-Nouri and Nilsson (2001) proposed that the basic SPT enhancement effect instead derives from increased self-involvement when enactment occurs at encoding. An alternative interpretation of encoding enhancement has been proposed by Noice and Noice (2007) in terms of the concept of embodied cognition, whereby language use is facilitated by concurrent activation of appropriate affordances of the physical environment (Glenberg \& Kaschak, 2002).

Were Kormi-Nouri and Nilsson (2001) correct to reject the hypothesis that enactment at retrieval can improve the accuracy of recall? Our consideration of this hypothesis was prompted by informal observation of some of the participants during testing for a recent study of spatial memory (Martin \& Jones, 2007). Prior to formally recording their responses, these participants spontaneously ranged their hands over the blank response templates. They appeared to believe, at least implicitly, that activating these motor patterns might lead to more accurate retrieval performance. Of course, it is alternatively possible that in reality these participants' motor activity had no effect on retrieval, or even interfered with it. However, consideration of the distinction between egocentric and allocentric spatial representations (Byrne, Becker, \& Burgess, 2007) does provide a theoretical rationale for the possible observation of an enactment effect at retrieval.

Byrne et al. (2007) have proposed that spatial memory is subserved by two forms of representation: an egocentric form tied to the organism's sensorimotor world and localized in the parietal and prefrontal areas of the brain, and an allocentric form organized independently of the organism's viewpoint and localized in the medial temporal

G.V.Jones, g.v.jones@warwick.ac.uk 
area. The necessary transformation between the two forms of representation is achieved along the circuit of Papez, whose integrity is known to be important for recollection (e.g., Aggleton \& Brown, 1999). Recall over the longer term in general relies on the allocentric type of representation. However, evidence from studies in which participants walk around a table to view objects from different angles suggests that such movement is linked to the updating of egocentric representations that also contribute to memory for the object array (Simons \& Wang, 1998; Wang \& Simons, 1999). The possibility to be explored here is that physical movement by the participant can serve in general to supplement reliance upon allocentric representations in recall by promoting a contribution from the other, egocentric component. Indeed, because of the widespread prevalence of motor imagery or activation without overt movement (e.g., Martin \& Jones, 1999; Szameitat, Shen, $\&$ Sterr, 2007), a contribution from this source is expected even in the absence of physical movement. According to this account, therefore, the adoption of relevant physical movement has the potential to enhance overall levels of memory retrieval and, conversely, the adoption of irrelevant physical movement has the potential to impair memory recall, due to the facilitation or impedance, respectively, of retrieval from egocentric representations. This prediction is investigated here primarily in the context of spatial memory linked to text messaging.

Following the development of the short message service (SMS) protocol, probably no other form of human behavior has increased in frequency in the last decade as much as has text messaging. In many countries, the number of younger people regularly texting has increased in a few years from no one to almost everyone. In the United States, for example, the volume of text messaging traffic has increased over the last 4 recorded years from 1.02 billion to 18.71 billion messages per month, and $25.2 \%$ of people 18-24 years of age now live in households without wireline telephones (Federal Communications Commission, 2008, pp. 7, 108). Crystal (2008) has recently explored the possible consequences of text messaging for a number of aspects of language use, including orthography and syntax. However, what is of present interest is the fact that texting exposes the texter regularly to a particular spatial configuration that maps the 26 letters of the alphabet onto the 10 numerical keys of the traditional phone. Can the texter's recall of this spatial array be improved by manipulating the way in which retrieval is instigated? In particular, what is the effect, if any, of first attempting to enact the movements that are required in order to send an actual text message?

Evidence against the existence of an enactment effect at retrieval in the SPT method cannot be viewed as decisive with regard to spatial learning in texting, in view of major differences between the two paradigms. SPT typically involves one-time presentation, within an intentional learning context. In contrast, spatial learning in texting occurs incidentally, over an extended period of practice. Accordingly, the study of texting also allows examination of how incidental learning is influenced by repeated exposure to stimulus information. There have been very few attempts to characterize the precise manner in which the probability of correct recall (as opposed to response latency) in incidental learning is influenced over a large number of repetitions. However, Anderson and colleagues (Anderson, 1982; Anderson \& Schooler, 1991) have proposed the existence of a power-law relation between the extent of learning and the number of opportunities to learn. This hypothesized relation may be written in the present case as $P\left(E_{T}\right)=B T^{-\alpha}$, where $P\left(E_{T}\right)$ is the probability of erroneous recall, $T$ is the number of textings, and $B$ and $\alpha$ are constants linked to initial performance and learning rate, respectively. Taking logarithms, $\ln \left[P\left(E_{T}\right)\right]=$ $\ln B-\alpha \cdot \ln T$, and thus the power law predicts that in a $\log -\log$ plot the probability of error should decline as a straight-line function of the number of learning opportunities. Deviation from the straight line has been widely observed for latency (rather than error) measures of performance (e.g., Newell \& Rosenbloom, 1981; Palmeri, 1997), but this deviation may be attributed to the fact that response latency cannot decline to zero. For response error, in contrast, a decline to zero is structurally possible, and therefore conformity or not to the power law is a matter of empirical investigation.

An experiment was carried out in light of these considerations. To investigate the potential influence of motor activation on the retrieval of spatial information acquired over the long term, the retrieval of both letter and number arrays was studied with or without preceding motor activity. When the participant was instructed to engage in movement (rather than inaction), this comprised either the enactment of a sequence that was relevant to the information to be recalled, or alternatively the performance of an irrelevant action that was incompatible with such enactment.

\section{METHOD}

\section{Participants}

These were 186 students (including 119 female participants), mean age 20.0 years (range, 18-24), tested individually or in small groups at the universities of Warwick and Oxford.

\section{Design}

Approximately equal numbers of participants were allocated to control and experimental motor conditions. In the former case, there were 95 participants in the inaction group. In the latter case, there were approximately equal numbers of participants in the action and enactment groups, with 44 and 47 participants, respectively.

\section{Procedure}

All participants received successive recall tests depicting a rectangular array of cells four units tall and three units wide. In a preliminary test to provide familiarization with the task, the cells were all blank, and participants were instructed to add all the numbers that would be found on a cell-phone keyboard, in their correct places. As expected, performance approached ceiling on this task and data were not formally analyzed. This was followed by recall of a letter array. For this, the 10 numerals were in their appropriate cells, and participants were instructed to add all the letters that would be found on a cell-phone keyboard, in their correct places. Finally, there was recall of a number array. For this, the cells were all blank, and participants were instructed to add all the numbers that would be found on a calculator keyboard, in their correct places. Starting from the top, the correct array for letters was / 1; $2 \mathrm{ABC} ; 3 \mathrm{DEF} / 4 \mathrm{GHI} ; 5 \mathrm{JKL}$; 
$6 \mathrm{MNO} / 7 \mathrm{PQRS} ; 8 \mathrm{TUV} ; 9 \mathrm{WXYZ} /$-; 0 ; - /, and for numbers was / 7; $8 ; 9 / 4 ; 5 ; 6 / 1 ; 2 ; 3 / 0 ;-;-/$.

Instructions for the three motor groups differed as follows. First, in the enactment condition, participants were instructed to tap out a specified sequence of responses (whose accuracy was not recorded) before they attempted to recall the array. The sequence took a different form for each of the three tasks, but in each case it was meaningful and familiar. In the preliminary task, the sequence was the phone number of a family member or friend; in the letter task, it was the text message how are you; and in the number task, it was the numeral 100. Second, in the action condition, participants were again instructed to start tapping before they attempted to recall each array, using their nonpreferred hands (i.e., left hand if the participant was right-handed, and vice versa), but not in relation to keyboard layout; for each type of task, participants were instructed to set up the same rhythm of groups of four similar taps with a brief gap between them. Third, in the inaction condition, participants received no instructions in relation to physical movement.

Following the recall tests, participants completed an inventory probing their text message and other usage. This included items on whether they texted; the duration for which they had been texting; their daily rate of sending texts; and whether they used a calculator either currently, or in the past but not currently, or never.

\section{RESULTS}

\section{Movement and Recall}

Figure 1 shows the accuracy of recall (i.e., the percentage of arrays recalled with complete accuracy) in this study for both the phone letter and calculator number arrays, in the three motor groups. For the recall of the letter array, there was a significant effect of motor condition $\left[\chi^{2}(2, N=186)=\right.$ $14.08, p=.001, \phi=.28]$. In pairwise comparisons, recall was significantly greater for the enactment condition than for the inaction condition $\left[\chi^{2}(1, N=142)=4.81, p<.05, \phi=\right.$ $.18]$, and significantly lower for the action condition than for the inaction condition $\left[\chi^{2}(1, N=139)=4.88, p<.05, \phi=\right.$ $.19]$. For the recall of the number array, there was again a significant effect of motor condition $\left[\chi^{2}(2, N=186)=19.77\right.$, $p<.001, \phi=.33]$. In pairwise comparisons, recall was significantly greater for the enactment condition than for the inaction condition $\left[\chi^{2}(1, N=142)=11.18, p<.001\right.$, $\phi=.28]$, but the difference between the action and inaction

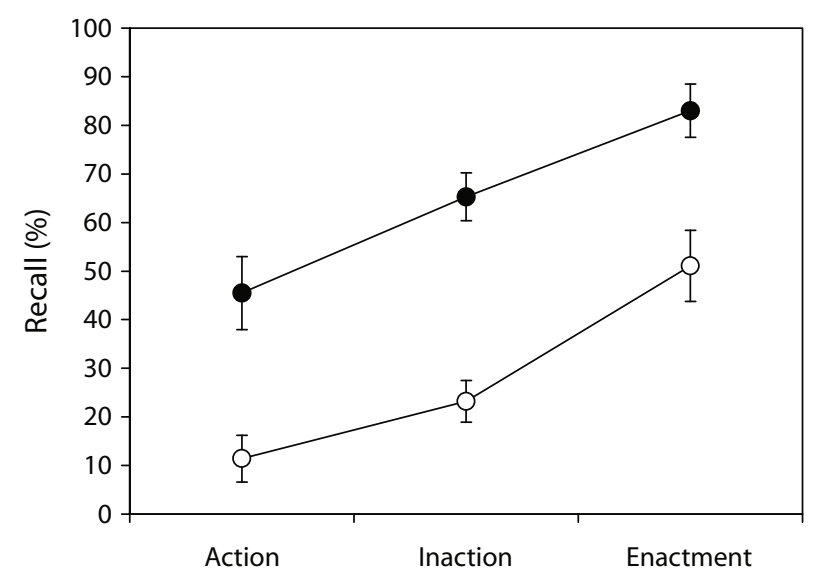

Figure 1. Recall of letter (solid) and number (hollow) arrays for action, inaction, and enactment motor conditions. Error bars indicate plus and minus one standard error of the mean (SEM).

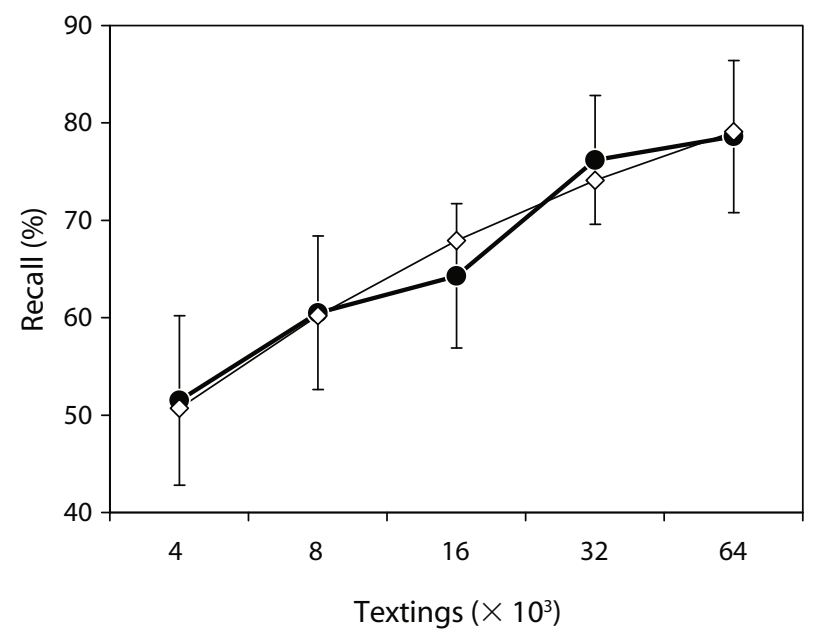

Figure 2. Recall of letter arrays (circles, with error bars indicating $\pm S E M$ ) as a function of numbers of textings sent, together with power-law predictions (diamonds).

conditions did not reach significance $\left[\chi^{2}(1, N=139)=\right.$ $2.67, p=.10]$.

\section{Effect of Texting Exposure}

All participants reported that they used text messaging. Mean text duration was 5.90 years $(S D=2.20)$ and mean text frequency was 7.70 messages per day $(S D=7.30)$. Number of textings was calculated as the product of texting duration (in days) and text frequency (per day), and participants were divided on this basis into five groups of approximately equal size (pooling across the three motor conditions to ensure adequate numbers of observations). The groups were defined by boundaries of up to 4,000, 8,000, 16,000, 32,000 , and $64,000+$ textings. Regression of $\ln \left[P\left(E_{T}\right)\right]$ on $\ln T$ revealed a significant linear trend $[F(1,3)=71.00$, $\left.p=.004, R^{2}=.96\right]$ with no evidence of a quadratic trend $[F(1,2)=0.09]$, in accordance with a power-law relation. The best-fitting equation was $\ln \left[P\left(E_{T}\right)\right]=1.855-$ $0.309 \ln T$, or $P\left(E_{T}\right)=6.39 T^{-0.309}$. Figure 2 shows the levels of observed letter recall together with the corresponding power-law levels - namely, $100\left[1-P\left(E_{T}\right)\right]$.

\section{Effect of Calculating Exposure}

The proportions of participants who had not used a calculator, had formerly used a calculator, and currently used a calculator were $2.7 \%, 49.2 \%$, and $48.1 \%$, respectively, and their overall levels of number array recall were $0 \%, 25.3 \%$, and $31.5 \%$, respectively. Because of its small size, the first group was combined with the second, prior to comparison with the third. The numerical difference in the level of recall between those who were not current users $(M=24.0 \%)$ and those who were $(M=31.5 \%)$ did not reach significance $\left[\chi^{2}(1, N=185)=1.30\right.$; power was $\left.98 \%\right]$.

\section{DISCUSSION}

The results of this study demonstrate that the accuracy of recall of previously acquired knowledge can be reliably influenced by controlling the nature of physical movement 
at the time of retrieval, at least for spatial information acquired incidentally over an extended period. Enactment of text messaging and calculator behavior led to significantly improved recall of, respectively, letter and number arrays. The enactment responses prior to recall were not recorded here, but the work of Rinck and Ellwart (2004) suggests that the familiarity of these sequences is important. They compared the entering on a blank keypad of a telephone number that was either familiar to the participant or not (it was provided by a yoked participant) and found that the accuracy of retrieving the location of the first key from memory declined significantly, from $80 \%$ in the former case to $50 \%$ in the latter.

The improvement in recall due to enactment that was observed here may be interpreted in terms of the hypothesis that appropriate physical movement promotes activation of egocentric representations, which, according to Byrne et al. (2007), are capable of supplementing the allocentric representations generally subserving longer term spatial memory. It was also found that irrelevant motor action exerted a significant effect in the direction opposite to enactment (though subject to a floor effect in the case of calculator numbers), which may be attributed to the disruption of motor activation that is present even in the absence of overt movement (e.g., Jeannerod, 1997; Martin \& Jones, 1999). It would be valuable to examine in an imaging investigation the hypothesis that enactment and irrelevant action exert opposing modulatory effects on activity associated with egocentric-allocentric transformation along the circuit of Papez (see also Aggleton \& Brown, 1999; Byrne et al., 2007). The present results are also consistent with the general principle of embodied cognition, insofar as they provide evidence for an interactionist account of the relation between movement and cognition (e.g., Noice \& Noice, 2007).

It is informative to consider the present results in the context of the subject-performed task, despite major procedural differences such as the SPT's use of intentional learning and verbal stimuli, and the circumstance that SPT studies in general "do not deal with . . memory of real actions experienced in everyday life" (Zimmer \& Cohen, 2001, p. 20). Kormi-Nouri and Nilsson (2001) argued that SPT enhancement is the consequence of increased integration within episodic memory produced by enactment at encoding only. However, they noted the finding of KormiNouri et al. (1994) that when participants were presented without enactment with phrases involving body parts (e.g., shake the head), and subsequently the verb (i.e., shake) was used as a retrieval cue for recall of the noun (i.e., head), then enactment of the verb at retrieval increased recall significantly, from $26 \%$ to $52 \%$ (see also Norris \& West, 1993). Engelkamp (2001) has pointed to further evidence of specifically motoric facilitation at retrieval. In particular, Engelkamp, Zimmer, Mohr, and Sellen (1994) found that recognition for action phrases (both conventional and unconventional-e.g., open the book and plant the hammer, respectively) was significantly higher when enactment occurred at both retrieval and encoding than when it occurred at encoding alone, as was also recognition of verbs denoting actions typically performed with two hands (e.g., peel). In addition, Engelkamp et al. found that recognition of verbs typically associated with a single hand (e.g., point), was significantly better when the hand that enacted at retrieval was the same as that which had enacted at encoding, rather than the participant's other hand (balanced over left and right hands), implicating relatively specific motoric processes in retrieval facilitation.

In a further SPT study, Mulligan and Hornstein (2003) found that although the recognition of a phrase such as knock on the door was better if enacted at encoding by the participant rather than by the experimenter, in both cases recognition was further improved if enactment was also carried out at retrieval (by the same person). Mulligan and Hornstein attributed these participant and experimenter retrieval effects to the roles of the participant's motor and visual systems, respectively. However, it is possible to hypothesize that even the latter experimenter retrieval effect (i.e., the beneficial effect of consistent observation by the participant) might have had a motor basis. Recent evidence suggests that not only mirror cell areas of the brain but also its motor system in general can be activated merely by the observation of appropriate movement (see, e.g., Tkach, Reimer, \& Hatsopoulos, 2007). Hence, motor activation may underlie not only the participant retrieval effect but also the experimenter retrieval effect in SPT observed by Mulligan and Hornstein (2003).

It was also found in the present study that, across participants, the overall level of error in letter recall decreased as a function of the number of learning opportunities in close conformity with the power law of learning proposed by Anderson and his colleagues (e.g., Anderson, 1982; Anderson \& Schooler, 1991). There was no significant evidence of asymptotic deviation from the power law, as has been observed previously (Martin \& Jones, 2007). In the present study, unlike the earlier one, the overall learning function was cumulated over three different motor conditions, and the present result therefore raises the possibility, in principle, that the power law may apply satisfactorily to the aggregated union of a set of subpopulations to which it does not apply individually; similar observations have been discussed by Wainer and Brown (2004), but have as yet received relatively little attention within psychology. More generally, the ongoing secular increase in the prevalence of texting was indicated by a participation rate in texting here of $100 \%$ (increased from the $83 \%$ reported by Martin \& Jones, 2007) and an average duration of texting of 5.9 years (increased from 3.3 years for those who texted; mean age in both studies was 20 years). No comparable effect was observed for calculator exposure, presumably reflecting either the relatively restricted range of calculator usage or a relatively uniform familiarity with the same numerical layout found on computer keyboards.

Finally, the present finding that people can improve the accuracy of their retrieval of a spatial array by engaging in appropriate physical enactment not only has theoretical relevance for the relation between allocentric and egocentric processes in recall, but also is potentially of practical relevance. For example, people frequently encounter problems in remembering PINs and other security codes. 
The present results suggest that the strategy of simulating the physical keying in of the PIN may serve to assist its correct retrieval. Furthermore, if the retrieval of such information from memory can indeed be helped by physical movement in this way, then interface designers might assist this process by maintaining high levels of consistency in spatial layout across different interfaces.

\section{AUTHOR NOTE}

The authors are grateful to S. Richardson, D. Russo, and B. Koperwas for help with data collection and transcription. Correspondence concerning this article may be addressed to G. V. Jones, Department of Psychology, University of Warwick, Coventry CV4 7AL England (e-mail: g.v.jones@warwick.ac.uk).

\section{REFERENCES}

Aggleton, J., \& Brown, M. (1999). Episodic memory, amnesia and the hippocampal-anterior thalamic axis. Behavioral \& Brain Sciences, 22, 425-444. doi:10.1017/S0140525X99002034

Anderson, J. R. (1982). Acquisition of cognitive skill. Psychological Review, 89, 369-406. doi:10.1037/0033-295X.89.4.369

ANDERSON, J. R., \& SCHOOLER, L. J. (1991). Reflections of the environment in memory. Psychological Science, 2, 396-408. doi:10.1111/j $.1467-9280.1991$. tb00174.x

Atkinson, R. C., \& Shiffrin, R. M. (1968). Human memory: A proposed system and its control processes. In K. W. Spence \& J. T. Spence (Eds.), The psychology of learning and motivation: Advances in research and theory (Vol. 2, pp. 89-195). New York: Academic Press.

Byrne, P., Becker, S., \& Burgess, N. (2007). Remembering the past and imagining the future: A neural model of spatial memory and imagery. Psychological Review, 114, 340-375. doi:10.1037/0033 $-295 X .114 .2 .340$

Chum, M., Bekkering, H., Dodd, M. D., \& Pratt, J. (2007). Motor and visual codes interact to facilitate visuospatial memory performance. Psychonomic Bulletin \& Review, 14, 1189-1193.

Crystal, D. (2008). txting: the gr $8 d b 8$. Oxford: Oxford University Press.

ENGELKAMP, J. (2001). What does it mean that the motor component is not crucial? Comments on Kormi-Nouri and Nilsson. In H. D. Zimmer et al. (Eds.), Memory for action: A distinct form of episodic memory? (pp. 144-150). New York: Oxford University Press.

Engelkamp, J., Zimmer, H. D., Mohr, G., \& Sellen, O. (1994). Memory of self-performed tasks: Self-performing during recognition. Memory \& Cognition, 22, 34-39.

Federal Communications Commission (2008). Twelfth report (FCC 08-28). Washington, DC.

GlenberG, A. M., \& KaschaK, M. P. (2002). Grounding language in action. Psychonomic Bulletin \& Review, 9, 558-565.

JEANNEROD, M. (1997). The cognitive neuroscience of action. Oxford: Blackwell.

Kormi-Nouri, R., \& NiLSSON, L.-G. (2001). The motor component is not crucial! In H. D. Zimmer et al. (Eds.), Memory for action: A distinct form of episodic memory? (pp. 97-111). New York: Oxford University Press.

Kormi-Nouri, R., Nyberg, L., \& Nilsson, L.-G. (1994). The effect of retrieval enactment on recall of subject-performed tasks and verbal tasks. Memory \& Cognition, 22, 723-728.

LogIE, R. H. (2003). Spatial and visual working memory: A mental workspace. In D. E. Irwin \& B. H. Ross (Eds.), The psychology of learning and motivation: Advances in research and theory. Cognitive vision (Vol. 42, pp. 37-78). San Diego: Academic Press.

Martin, M., \& Jones, G. V. (1999). Motor imagery theory of a contralateral handedness effect in recognition memory: Toward a chiral psychology of cognition. Journal of Experimental Psychology: General, 128, 265-282. doi:10.1037/0096-3445.128.3.265

Martin, M., \& Jones, G. V. (2007). Asymptotic learning of alphanumeric coding in autobiographical memory. Cognition, 102, 311320. doi:10.1016/j.cognition.2006.02.001

Mulligan, N. W., \& Hornstein, S. L. (2003). Memory for actions: Self-performed tasks and the reenactment effect. Memory \& Cognition, 31, 412-421.

Newell, A., \& Rosenbloom, P. S. (1981). Mechanisms of skill acquisition and the law of practice. In J. R. Anderson (Ed.), Cognitive skills and their acquisition (pp. 1-55). Hillsdale, NJ: Erlbaum.

NiLsson, L.-G. (2000). Remembering actions and words. In E. Tulving \& F. I. M. Craik (Eds.), The Oxford handbook of memory (pp. 137148). New York: Oxford University Press.

Norce, H., \& Noice, T. (2001). Learning dialogue with and without movement. Memory \& Cognition, 29, 820-827.

Noice, H., \& Noice, T. (2007). What studies of actors and acting can tell us about memory and cognitive functioning. Current $D i-$ rections in Psychological Science, 15, 14-18. doi:10.1111/j.0963 -7214.2006.00398.x

NorRIs, M. P., \& WeST, R. L. (1993). Activity memory and aging: The role of motor retrieval and strategic processing. Psychology \& Aging, 8, 81-86. doi:10.1037/0882-7974.8.1.81

PALMERI, T. J. (1997). Exemplar similarity and the development of automaticity. Journal of Experimental Psychology: Learning, Memory, \& Cognition, 23, 324-354. doi:10.1037/0278-7393.23.2.324

RincK, M., \& ElLWART, T. (2004). Repetition priming during the use and recall of numerical keypads. European Journal of Cognitive Psychology, 16, 841-861. doi:10.1080/09541440340000376

SimONs, D., \& WANG, R. (1998). Perceiving real-world viewpoint changes. Psychological Science, 9, 315-320. doi:10.1111/1467-9280.00062

Szameitat, A. J., Shen, S., \& Sterr, A. (2007). Motor imagery of complex everyday movements. An fMRI study. NeuroImage, 34, 702-713. doi:10.1016/j.neuroimage.2006.09.033

TKaCh, D., Reimer, J., \& Hatsopoulos, N. G. (2007). Congruent activity during action and action observation in motor cortex. Journal of Neuroscience, 27, 13241-13250. doi:10.1523/JNEUROSCI.2895-07.2007

WAINER, H., \& Brown, L. M. (2004). Two statistical paradoxes in the interpretation of group differences: Illustrated with medical school admission and licensing data. American Statistician, 58, 117-123. doi:10.1198/0003130043268

WANG, R., \& Simons, D. (1999). Active and passive scene recognition across views. Cognition, 70, 191-210. doi:10.1016/S0010-0277(99) 00012-8

Zimmer, H. D., \& Cohen, R. L. (2001). Remembering actions: A specific type of memory? In H. D. Zimmer et al. (Eds.), Memory for action: A distinct form of episodic memory? (pp. 3-24). New York: Oxford University Press.

(Manuscript received August 3, 2008; revision accepted for publication January 14, 2009.) 